\title{
La remodelación de El Periódico de Catalunya: hacia el modelo de prensa de servicios
}

\author{
Dr. Aintzane Alberdi Ezpeleta \\ Dr. José Ignacio Armentia Vizuete \\ Dr. José María Caminos Marcet \\ Dra. Flora Marín Murillo ${ }^{1}$
}

\section{RESUMEN}

A lo largo de la última década del siglo XX el modelo de diario de servicios ha ido consolidándose en España con unas características, tanto formales como relativas a los contenidos, propias y diferenciadoras con respecto a los modelos predominantes anteriormente, fundamentalmente el informativo-interpretativo y las fórmulas híbridas "informativo-opinión" $e$ "informativo-sensacionalista”. El profundo rediseño -que ha afectado a todos los aspectos del medio-protagonizado a lo largo del año 2000 por El Periódico de Catalunya, de Barcelona, puede ser tomados en consideración como claro exponente del asentamiento del nuevo modelo de diario, tal y como se intenta demostrar en este trabajo. Antes de su rediseño, el espacio dedicado por El Periódico a los temas de servicios suponía un 55\% del total, una cifra que pasa al 71,5\% tras el cambio de maqueta. Este artículo se basa en una investigación financiada por la Universidad del País Vasco.

\section{ABSTRACT}

Throughout the last decade of century $X X$ the newspaper model of services has been consolidated in Spain with own characteristics, as much formal as relative to the contents, and differentiating with respect to the predominant models previously, fundamentally informative one and the hybrid formulas "information-opinion" and "informative-popular". The deep redesigns suffered by El Periódico de Catalunya from Barcelona -which had happened during 2000 yearcan be taken in consideration like a clear exponent of the establishment of the new newspaper model, so and as it is tried to demonstrate in this work. In this redesign, the presence of the subjects of services has been increased remarkably: before redesigning, the subjects of services occupied a 55\% of the total space to fill; after it, this average increased until 71,5\%. This article is based in a reserarch work financed by the University of the Basque Country.

Palabras claves: Periodismo/Servicios/El Periódico de Catalunya.

Key words: Journalism/Services/El Periódico de Catalunya.

1 Aintzane Alberdi es una periodista especializada en temas de servicios. José Ignacio Armentia, José María Caminos y Flora Marín son profesores de la Facultad de Ciencias Sociales y de la Comunicación de la Universidad del País Vasco 
La remodelación de El Periódico de Catalunya: hacia el modelo de prensa de servicios

\section{Qué se entiende por periodismo de servicios}

n plena era de la globalización cada día vivimos más pendientes de
nosotros mismos y nuestros destinos. Frente a la proliferación de orga-
nizaciones no gubernamentales y solidarias, nuestro interés por los
problemas del vecino se ha visto frenado por un aislacionismo social que nos lleva a buscar al otro en las páginas de corazón, en el cotilleo absurdo que antes estaba reservado al inquilino de al lado. «La autoconciencia ha sustituido a la conciencia de clase, la conciencia narcisista sustituye la conciencia política, sustitución que no debe ni mucho menos interpretarse como el eterno debate sobre la lucha de clases», dice Lipovetsky (2000:65). En este mirarnos el ombligo se inscribe ese entusiasmo por el conocimiento de uno mismo y su realización personal.

Nuestra sociedad es una sociedad aparentemente contradictoria plagada de lógicas «irreconciliables». Abrumados por las informaciones y los acontecimientos que los medios de comunicación nos proporcionan con avidez y prontitud, nuestra sensibilidad renace y muere cada día con una nueva información. La respuesta inmediata,- donaciones, firmas,- sustituye a una toma de conciencia profunda.

Este modelo de sociedad estaría conformado por infinidad de microcolectividades heterogéneas entre sí. El individuo actual renuncia en general a discutir sus opiniones: «vive y deja vivir». Del mismo modo renuncia a buscar un sentido único y totalizante para la vida. Ésta es una postura confortable, alérgica a las exigencias radicales.

Sin embargo, y curiosamente, esta postura vital, en palabras de Lipovetsky (2000:53) no nacería de una concienciación desencantada , sino más bien de «una lógica social individualista hedonista impulsada por el universo de los objetos y los signos, y de una lógica terapéutica y psicológica elaborada desde el siglo XIX a partir del enfoque psicopatológico».

La publicidad, cómo no, se hace receptora y emisora de este nuevo individuo, y nos vende libertad, seguridad en uno mismo, confianza, etc., a través de los objetos, símbolos del progreso tecnológico y social. El discurso enunciado busca al individuo consumidor, y éste consume ideales personales entre coches, teléfonos móviles y buscadores de internet.

Al igual que la publicidad, la prensa está considerada, según Fontcuberta (Reflexiones Académicas, $\mathrm{n}^{\circ} 12,2000$ ) «cada vez más como objeto de consumo, no siempre vinculada con la noticia/información de actualidad», por lo que ofrece a este individuo narcisista un producto que satisfaga su ego personal y sus necesidades, sean inmediatas o no.

Es en este contexto, y ligado a esta sociedad, donde podemos hablar de un cambio en los medios y sobre todo en la prensa. La adaptación del medio a este nuevo individuo implica satisfacer a un renovado consumidor que solicita de la prensa no sólo información, que por otra parte ya recibe a través de otros canales, sino y, sobre todo, herramientas útiles para su bienestar personal. 
El diario de servicio viene a llenar ese hueco donde el periódico, aún manteniendo su función originaria, seduce al lector a través de su diseño, de la pedagogía de sus imágenes, de las informaciones que buscan la «verdad» y su bienestar. Cada uno es responsable de su propia vida, de su capital estético, afectivo o psíquico, el diario de servicio esta ahí para ayudarnos administrar este capital. Es en este nuevo concepto de medio donde, como dice Lipovetsky, la desocialización y la socialización se identifican. Al final de este desierto social nos encontramos al individuo soberano, libre, informado y prudente. Este proceso de personalización nos lleva hacia una nueva forma de socialización donde el individuo se integra gracias a la persuasión invocando salud, seguridad, y racionalidad.

El diario de servicio se fundamenta básicamente en dar al consumidor lo que éste desea y éste desea llenar su tiempo libre, saber cómo llegar a la farmacia de guardia más próxima, conocer la forma en que los avances científicos pueden afectar directamente a su salud o, simplemente, estar al corriente de los problemas de infraestructura a los que se va a enfrentar los próximos veinte días a la hora de ir a trabajar.

Sin embargo, y como veremos, este tipo de diario tiene su partida de nacimiento en Estados Unidos, y sólo en los últimos años podemos hablar de su establecimiento en España. En esta larga travesía, el diario de servicio norteamericano se ha ido impregnando de diversas particularidades que tienen mucho que ver con la tradición de la prensa en Europa, y las propias peculiaridades de una sociedad que a pesar de la globalización todavía conserva algunos rasgos autóctonos.

La historia del periodismo es el punto de partida para el estudio y análisis de la evolución de las ciencias sociales en general, y del periodismo como ciencia social en particular. Sólo desde el conocimiento de las distintas formas de hacer periodismo que se han correspondido con diferentes fases históricas, podemos analizar con coherencia la evolución del periodismo y, desde esa perspectiva, estudiar en la actualidad las posibles evoluciones futuras que sobre el quehacer periodístico se ciernen desde estos primeros años del siglo XXI.

La etapa histórica de lo que entendemos por periodismo moderno arranca a mediados del siglo XIX. Autores como Angel Benito (1982:74) sitúan el inicio de esta etapa en el año 1850, fecha alrededor de la que podemos ubicar la primera industrialización. A lo largo de esta época, que se corresponde con el desarrollo del parlamentarismo, se cultiva una prensa escasamente informativa en la que predominan los comentarios de opinión, fundamentalmente políticos, y que acaban por estructurar una etapa que conocemos hoy como de periodismo ideológico.

En las dos últimas décadas del siglo XIX comienzan a tomar cuerpo nuevos enfoques en el trabajo periodístico. Desde 1870 y aproximadamente hasta 1920 coexisten estas dos formas de hacer periodismo: el periodismo ideológico y el 
periodismo informativo. Finalizada la Primera Guerra Mundial y fundamentalmente alrededor de 1920 se puede decir que el periodismo informativo desplaza al ideológico y acaba por imponerse en casi todos los países industrializados occidentales. A lo largo de unos 30 años, desde 1920 hasta 1950, el periodismo informativo alcanza su máxima implantación, de la mano de los rapidísimos cambios tecnológicos que se incorporan a la producción periodística. En esta fase se desarrollan también muy variadas formas de expresión lingüística para relatar los hechos o lo que conocemos como noticias.

Este proceso que va desde el auge del periodismo ideológico, pasa por la coexistencia con el periodismo informativo y finaliza con la implantación de éste último, se configuró, en palabras de Josep María Casasús (1991:22), «a partir de la combinación y de las influencias entre diversos factores tecnológicos, económicos, políticos, profesionales, estilísticos y, en definitiva, comunicativos».

Desde el final de la Segunda Guerra Mundial el periodismo moderno entra en una tercera etapa que se caracteriza por la profundidad en el tratamiento temático. La aparición de la radio y la televisión y su implantación como medios para la difusión de informaciones, obliga a la prensa escrita, con una menor inmediatez, a hacer un esfuerzo especial para la difusión de sus mensajes periodísticos. A la rapidez que ofrece la radio y la televisión, la prensa escrita opone la profundidad y la reflexión. Es el momento del periodismo explicativo o interpretativo, en el que el relato de los hechos se combina con las interpretaciones que el periodista hace de ellos. Es la implantación de géneros que utilizan de forma combinada el relato con el comentario. Es una etapa que se conoce con el nombre de periodismo de explicación o periodismo de profundidad. El desarrollo tecnológico de esta época permite la consolidación de la fotografía, que pasa de ser un mero material de apoyo al texto a un género con todas sus cualidades específicas. Otro tanto ocurre con la infografía, que combina a la perfección la imagen textual y la icónica y las ensambla para consolidar un nuevo género, el infoperiodismo, que alcanza su despegue en los últimos años del siglo XX.

A estas tres grandes etapas de la historia del periodismo les corresponden también distintas formas de expresión y comunicación. Unas son meramente lingüísticas, es decir, utilizan el lenguaje escrito para la difusión de mensajes periodísticos (texto escrito); otras son no lingüísticas, es decir, utilizan códigos icónicos para la difusión de mensajes (fotografía); y unas terceras son paralingüísticas, es decir, provienen de la combinación de los dos lenguajes anteriores (infoperiodismo).

$\mathrm{Al}$ comienzo de la década de los 80 se produce un cambio importante en la tendencia de los modelos de diario imperantes por esas fechas. En setiembre de 1982 salió al mercado el diario norteamericano USA Today. Su nacimiento supuso un considerable cambio al tipo de periodismo que se venía practicando por esa época y puso los cimientos de una concepción nueva del periodismo, tanto desde 
el punto de vista temático como en lo que se refiere a su presentación formal. Además, marcó una nueva tendencia en el quehacer periodístico que fue rápidamente aceptada por el público lector. USA Today pasó a convertirse en pocos años en el diario estadounidense de información general más vendido. Durante el primer semestre de 1999 su difusión media fue de 1.739.294 ejemplares durante los días de la semana, y de 2.133.467 durante los domingos.

Con el USA Today comienza a implantarse un nuevo concepto del periodismo, fundamentalmente en una doble vertiente. Por un lado, tal y como afirman Armentia, Elexgaray y Pérez (1999:74) «aparece un nuevo concepto en relación a la presentación estética de la prensa escrita: el «diseño visualizante». Se trata de un diseño que recoge en sus aspectos formales algunas de las características de los medios audiovisuales. Entre las más importantes podríamos destacar: el empleo de color, la proliferación de fotografías, gráficos e ilustraciones y la variada y rica tipografía que emplea en sus páginas». Por otro lado, apuesta por una concepción nueva también en el tratamiento textual. Gran variedad de informaciones, más bien breves, y una clara apuesta por las informaciones de servicios.

Podemos decir, incluso, que desde la aparición de este periódico estamos asistiendo a uno de los cambios más radicales que hay en lo que a los modelos de diarios se refiere. Tal y como afirma Josep María Casasús (1991:23), «el modelo representado, todavía incipientemente, por el USA Today se aparta sustancialmente de la concepción hemerográfica materializada en modelos como The Washington Post, The Times o The New York Times, como diarios informativo-interpretativos, pero también de The Sun, The Daily Mirror, The Daily Express o Bild Zeiutung, como diarios de modelo genuino popular-sensacionalista».

Estamos, pues, ante un nuevo modelo de diario, propio de estas dos últimas décadas del siglo $\mathrm{XX}$, que no se ajusta a los parámetros propios de la prensa de calidad informativo-interpretativa, pero tampoco a las líneas maestras marcadas por la prensa sensacionalista. Un nuevo modelo que a partir de la década de los 80 comienza a perfilar lo que se entiende como periodismo de servicios.

Muchos y muy variados han sido los elementos que han confluido para la implantación de este nuevo modelo de diario. Los cambios sociales que se han producido en los últimos años en la dirección de consolidar lo que se ha conocido con el nombre de sociedad del bienestar y el consiguiente desarrollo de la cultura del ocio; el estancamiento de los índices de lectura de prensa escrita; los importantísimos cambios tecnológicos aplicados también, cómo no, a la producción periodística; los cambios en el interés temático operado por los lectores; la influencia del mundo audiovisual en las nuevas audiencias que se van incorporando a la lectura de prensa escrita, etc., son todos ellos elementos que subyacen en las nuevas estrategias que va adoptando la prensa escrita desde hace ya más de dos décadas.

El nuevo modelo de diario que se va ya apuntando con claridad a lo largo de los últimos años, conserva, como no podía ser de otra forma, las características 
más importantes del modelo imperante desde los años 50. Un modelo marcado por la profundidad y el análisis; la interpretación y el comentario unido al relato de los hechos. Un periodismo temáticamente vinculado a los temas políticos, internacionales, etc., y que acababan por definir lo que se ha entendido dentro del marco de la prensa de calidad como el modelo informativo-interpretativo, propio del periodismo de profundidad.

En la situación actual, como ha sucedido en cualquiera de las etapas por las que históricamente ha atravesado el periodismo, conviven las nuevas formas que aquí estamos apuntando con lo que es el ejemplo más genuino de la etapa anterior.

En estos momentos, podemos decir, pues, que nos encontramos en una fase de clara transición caracterizada por el hacer periodístico de los últimos años del siglo XX, un periodismo informativo-interpretativo, que va cediendo terreno poco a poco a unas nuevas formas periodísticas que no tienen todavía un corpus cerrado, pero que se caracteriza por la atención que presta a nuevas temáticas, nuevas fuentes, nuevos enfoques, nuevas presentaciones y formas de expresión, todo ello orientado a ofrecer un mayor servicio al lector y que identificamos con el nombre de periodismo de servicios o, en palabras de Josep María Casasús, como «periodismo social».

Entre las características más importantes de este nuevo modelo de diario podríamos destacar las siguientes:

1. Información y opinión: El modelo actual de periódico de servicios es el de un diario preocupado por la correcta clasificación y el orden de la información. Al igual que sucede con el modelo informativo-interpretativo, el periódico procede a una separación rigurosa de los géneros periodísticos encaminada a diferenciar razonablemente la opinión de los simples relatos de hechos.

2. Diseño y compaginación: Desde el punto de vista formal posee un planteamiento abierto al impacto visual. Se ha preocupado en utilizar con toda su extensión las posibilidades de las nuevas tecnologías en las artes gráficas, de ahí que no dude en utilizar el color, los dibujos, gráficos, despieces y todo tipo de recursos que fortalezcan el impacto visual encaminado a facilitar la lectura de «doble velocidad». A pesar de ello, prevalece también la jerarquía informativa y se observa con facilidad la importancia que el medio dedicada a cada una de sus informaciones. También existe proporción entre el volumen de informaciones y las columnas utilizadas para la titulación, así como el número de columnas que ocupa un titular con el tamaño del mismo.

3. Ordenación temática: Al igual que en el modelo informativo-interpretativo, los diarios de servicios siguen un esquema en el que los materiales informativos aparecen ordenados de acuerdo con un plan lógico y racional. Es decir, el esquema de las secciones se mantiene de tal forma que llega a constituirse en un elemento fundamental de la fisonomía del periódico. Un criterio de ordenación, además, en el que claramente predomina la ordenación temática frente a la geo- 
gráfica. Lo que sucede es que el nuevo modelo modifica la designación de las secciones con unos nombres que dejan ver a las claras las temáticas predilectas del nuevo modelo. Así, las nuevas secciones de estos medios pasan a denominarse: Ciudadanos, Vivir, Actualidad, Cosas de la Vida, etc...

4. Los elementos tipográficos: El diario de servicios se caracteriza por una mayor libertad en la utilización de los elementos tipográficos. Se utilizan recursos llamativos, tales como las tramas en color, diversos tipos de letra, etc. Sin embargo, estos juegos tipográficos se hacen dentro de un orden, sin caer en la expresividad máxima de los diarios sensacionalistas y buscan también el orden, la proporción y el equilibrio.

5. El uso del material gráfico: La utilización de la fotografía en este tipo de modelo es muy selectiva. Menos pero más grandes fotografías para resaltar la importancia de un elemento gráfico que ya no es solamente el apoyo del texto. Además, se caracteriza por la utilización de una gran variedad de recursos visuales: fotografías, infográficos, gráficos, tablas, etc. Todos ellos conjuntados para buscar una mayor expresividad en los textos de servicios.

6. Estilo de redacción y temáticas: Mantiene la precisa diferenciación de los géneros periodísticos, propia del modelo informativo-interpretativo. El estilo de redacción respeta la división fundamental de los géneros informativos y los de opinión, pero manifiesta una tendencia a la personalización de los materiales informativos.

Por lo que respecta a las temáticas, mientras que el modelo informativointerpretativo pone su punto de mira en las informaciones, políticas, sociales y económicas, es decir, lo que entendemos por noticias de interés público, un abanico temático que deriva de la pertenencia de las personas a un ámbito de convivencia social común, el modelo de periodismo de servicios, haciendo hincapié también en las temáticas de interés público, se orienta también hacia los temas directamente relacionados con el día a día de la vida de las personas y que tienen una relación más directa con el interés psicológico de las personas, o lo que entendemos por interés del público.

\section{Periodismo de servicios en España}

Tras el rediseño de La Vanguardia y la llegada de los 90 cambia radicalmente la tendencia que dominaba en el Estado español durante la década anterior, y entra en escena la escuela anglosajona de diseño periodístico con un afán decidido de transformar los criterios establecidos durante años.

Tal y como indica Casasús, "desde el primer proyecto de rediseño, el elaborado por el estudio de Nueva York de Walter Bernard/Milton Glaser para la renovada La Vanguardia, pasando por el trabajo de Roger Black para El Sol, y terminando con la aportación de Louis Silverstein para El Observador, toda la impronta de una nueva escuela irrumpe en el panorama de la imagen de la prensa 
y en poco tiempo empieza a ser imitada. Se amplían los criterios de trabajo: la tipografía y las familias de letras cobran otro valor, un sentido gráfico diferente" (Casasús y otros, 1991, 36).

Los esquemas que habían permanecido invariables durante años y que parecían inamovibles "se modifican de tal manera que ahora las fotografías interrumpen la lectura de titulares y textos y los sumarios/destacados aparecen en el centro de los cuerpos de texto, rompiendo la monotonía de los bloques grises. Casi todo se hace con otras reglas, con criterios que se defienden desde puntos de vista que abogan por una mayor facilidad para la lectura, en una sociedad que cada día tiende a leer menos. Se da más trascendencia en las redacciones a la figura del diseñador. Se crean departamentos de arte. Se potencian las portadas, los suplementos y los magazines dominicales, con mayor aportación de los grafistas. Nacen secciones de infografismo con peso específico en las salas de redacción, como las de El Mundo o La Vanguardia. Se cuidan, con todo lujo de detalles, los coleccionables que se entregan con las ediciones de los domingos. Parece, como concecuencia de lo descrito, que el protagonismo de la forma empieza a equilibrarse con el que ha tenido durante más de cien años el fondo, el contenido. Las nuevas tecnologías, especialmente en lo que se refiere a la impresión en offset color, a la informática y a la microelectrónica, están siendo las grandes aliadas de este fenómeno que se está detectando en el mundo de la prensa. Ello hace que las empresas periodísticas y las redacciones de los diarios empiecen a valorar todo lo que supone la buena presentación de un medio escrito en un mundo en el que la imagen cada día adquiere mayor fuerza" (Casasús y otros, 1991, 36 y 37).

Junto a la influencia de diseñadores norteamericanos de la talla de Milton Glaser, Walter Bernard, Roger Black o Louis Silverstein; distintos diseñadores españoles han irrumpido con fuerza en el mercado de la prensa configurando propuestas formales alternativas a la maqueta de inspiración centroeuropea elaborada por Reinhard Gäde para El País. Entre estos diseñadores españoles cabría destacar los nombres de Antoni Cases, Ricardo Bermejo, Alberto Torregrosa, Enric Satué, etc.

La apuesta decidida por el color es una de las características de los rediseños que se producen a lo largo de los 90. Tal y como acertadamente apuntaba Bernardo Díaz Nosty (1992, 55), tan sólo en el período 1990-92 medio centenar de diarios españoles afrontó un proceso de rediseño. Esta tendencia a la renovación se mantendría en los años siguientes. En muchas ocasiones estos procesos de rediseño fueron parejos a la adquisición de nueva tecnología de impresión $-A B C$, El Correo Español, La Voz de Galicia...-. En otras ocasiones el rediseño ha sido más profundo y ha afectado a aspectos tales como la tipografía, estructura general de la publicación, etc...

A pesar de que los cambios formales de la inmensa mayoría de los diarios del Estado evolucionan de forma lenta, la nueva demanda informativa que ha 
generado en los lectores la llamada "sociedad del bienestar" tiene cada vez más reflejo en el contenido de los periódicos del nuevo siglo. Los temas de salud, ecología, ocio, deporte, etc., comienzan a estar presentes tanto en sus páginas especiales monográficas como en las secciones habituales. Además, en ocasiones, en el tratamiento de algunos temas se aprecia una cierta influencia del nuevo enfoque de "periodismo de servicios". Aunque todavía no existe esa conciencia plena de servicio al lector, por medio de la cual todas las informaciones deberían ir enfocadas a ofrecer unos datos de interés práctico.

En realidad, la ampliación de la oferta informativa hacia esa serie de asuntos que cada vez atraen más a los lectores queda más reflejada en los múltiples cuadernillos monográficos y suplementos de fin de semana que en las secciones habituales de los diarios, donde la información política cubre aún la mayor parte de su superficie. A pesar de todo, la evolución queda patente: durante la segunda mitad de la década de los 90 todos los diarios han reforzado sus secciones de "Servicios".

Se podría afirmar que los aspectos más destacados del estilo con el que los diarios del Estado español afrontarán el nuevo milenio se centrarán en conseguir unos periódicos con una presentación clara y más directa, con textos cada vez más cortos y mejor escritos (muy pocos son en la actualidad los diarios que emplean directamente las noticias de agencia, sin trabajarlas). En general, se elaboran más los escritos para ofrecer una información más fácil de digerir, descompuesta en abundantes despieces, con los que el maquetador puede jugar en combinaciones que hacen más agradable la lectura. Afortunadamente cada vez hay más conciencia de que el diseño comienza en las salas de redacción.

Será cada vez más abundante el empleo de recursos gráficos que busquen una presentación más funcional y mejor señalizada, en la que se apueste por el uso del color y a través de la cual se trate de conectar con los nuevos hábitos de los lectores. Todo ello integrado en una nueva estructuración fragmentada, basada en la utilización de los denominados "formatos de lectura rápida" que garantizan la diversidad de ritmos de lectura.

Si los responsables de los diarios fueran más permeables a los gustos de los lectores que quedan de manifiesto en los diversos estudios realizados sobre las preferencias del público lector, tanto la tipografía como el resto de los recursos formales deberían ser usados cada vez de un modo más creativo, que permitan un margen de juego estético a los diseñadores, ofreciendo composiciones que traten de ajustar el diseño a las necesidades de la información y no al revés, tal y como ya se ha comenzado a hacer en las redacciones de algunos rotativos.

Dentro de esta filosofía habría que situar los recientes rediseños (marzo de 2000) de El Correo Español-El Pueblo Vasco y el de El Periódico de Catalunya (octubre de 2000). Ambos diseños, que responden a las características formales que venimos apuntando para la prensa de servicios, van a ser estudiados con detenimiento en las próximas páginas. 
Otros diarios de tiradas más modesta han seguido el mismo camino. Ya se ha apuntado con anterioridad el caso de El Correo de Andalucía, que el 16 de mayo de 2000 estrenaba nueva maqueta, obra del estudio de Antoni Cases. La filosofía del nuevo diseño es la de facilitar la lectura de la publicación. Con dicho fin se ha incrementado el cuerpo e interlineado de los textos que han pasado de un 9,5/10,3 a un 10/11,2. Asimismo, se ha modificado la composición de las páginas. Con la nueva maqueta se intenta que haya una información por página con sus correspondientes despieces y textos de apoyo. Cabe apuntar que en el diseño anterior era frecuente la existencia de tres o cuatro informaciones por plana.

También el grupo Joly ha rediseñado los periódicos Diario de Cádiz, Diario de Jerez y Europa Sur para unificar su formato con el de Diario de Sevilla. El grupo ha creado una gran redacción central en Sevilla, que es la encargada de elaborar la información nacional, internacional y de economía que comparten los cuatro diarios. Asimismo, Diario de Jerez y Europa Sur han modificado totalmente su cabecera, al igual que Diario de Cádiz, aunque éste en menor medida.

En el País Vasco, las nuevas tendencias han tenidos su reflejo en los recientes rediseños de Deia y Euskaldunon Egunkaria. Deia, un periódico nacido en Bilbao en junio de 1977, una semana antes de las primeras elecciones tras la muerte de Franco, estrenó diseño el 20 de octubre de 2000. La nueva maqueta fue obra del estudio de Ricardo Bermejo -Bega Comunicación-. El nuevo periódico se divide en tres partes. Por un lado, se encuentran las secciones "clásicas" (Metro, Euskadi, Opinión, Mundo, Economía, etc.). Por otro lado está la sección de Deportes, con un diseño muy arrevistado y una portada específica a todo color.

Por último, y ésta es una de las principales novedades del nuevo diseño, se sitúa el "D2, La revista de Deia", doce páginas dedicadas a los servicios, el ocio, la cultura y los espectáculos, en un formato a medio camino entre el "Vivir" de La Vanguardia y el "SOS" de Diario 16. Además, dicha sección, que también cuenta con una portada a todo color, incluye diariamente un reportaje en profundidad sobre diversas temáticas. Refiriendose a esta nueva sección, los reponsables de la publicación explicaban en un suplemento especial dedicado al nuevo diseño que se publicó el 19 de octubre de 2000 lo siguiente: "En Deia hemos pensado que el cierre del diario es un espacio privilegiado para provocar de nuevo el interés. En este sentido, D2 hará su presentación todos los días con una portada arrevistada en la que se conjugan elementos propios de una revista y de un periódico. (...) El tema de apertura de D2 va a ser, desde mañana, nuestra apuesta diaria de lectura en profundidad. Nada de política ni de políticos: al contrario, contenidos de interés humano y cívico. La salud, la educación, los avances científicos, el medio ambiente, el ocio y la televisión". En dicha sección se integran también los servicios: el tiempo, la cartelera, la agenda y los pasatiempos.

Otro diario del País Vasco que se rediseñó a lo largo de 2000, concretamente su nueva maqueta apareció el 6 de diciembre, coincidiendo con el décimo 
aniversario del nacimiento de la publicación, fue Euskaldunon Egunkaria. Este diario es el único del País Vasco que se edita íntegramente en euskara y tradicionalmente ha tratado de buscar fórmulas de presentación muy visuales -cuerpos de letra más grandes, interlineados en el texto más altos- para facilitar al máximo la lectura de sus páginas.

El nuevo diseño -obra de Andrés Gostin, Maixa Olano y Allende Arnaizincluye diversos formatos de lectura rápida, tales como titulares de múltiples elementos, sumarios y textos de apoyo. Asimismo, al inicio de cada sección se incluye una portadilla con una serie de ventanas con los temas más destacados incluidos en la misma. Por otra parte, sin desatender el estilo fundamentalmente informativo del rotativo, se han reforzado secciones como Servicios, Gente, Cultura, Agenda (5 páginas diarias) y Radio y Televisión.

\section{El rediseño de El Periódico de Catalunuya}

\subsection{Cambios formales}

El Periódico de Catalunya ha sido el primer diario del Estado -y uno de los primeros de Europa- en optar por editar la totalidad de sus páginas en color. Ello ha sido posible gracias a la puesta en funcionamiento de una moderna planta de impresión en la localidad barcelonesa de Parets de Vallès.

La entrada en funcionamiento de la nueva rotativa conllevó un rediseño de la publicación, que fue realizada por el estudio Cases i Asociats y en el que destaca -al margen de una ligera reducción en el formato- el empleo del color como un elemento fundamental en la confección del diario. De hecho, se ha asignado un color diferente a cada sección, para conseguir un diario visualmente más atractivo y ayudar al lector a identificar mejor cada sección.

El nuevo diseño salió a la calle el 3 diciembre de 2000 y, junto a la reducción de un centímetro en la anchura del rotativo, el mismo provocó el paso de una pauta de 6 columnas a otra de 5. Cabe señalar que El Periódico era una de las escasas publicaciones del Estado que seguía manteniendo una pauta de seis columnas frente a la tendencia generalizada de emplear cinco.

La tipografía de El Periódico también sufrió cambios. Frente a la omnipresencia de la Helvética, la Swift, una letra con remates, ha comenzado a utilizarse en los textos. Para dar más claridad a éstos, ahora se emplea un cuerpo de 9,8 puntos sobre un interlineado de 11,4 puntos. En los titulares, subtítulos y antetítulos se sigue empleando la Helvetica, aunque en una versión más ancha que en la etapa anterior. Al igual que sucedía con anterioridad, se mantiene el uso de pictogramas para situar rápidamente al lector sobre el contenido de la noticia que va a leer.

La nueva confección divide El Periódico en dos cuerpos diferenciados ambos van grapados, algo que hasta ahora sólo hacía $A B C-$. El primero de los cuadernillos está compuesto por El Tema del Día y tres bloques que agrupan las 
diferentes secciones: Internacional, Política y Economía. El segundo bloque corresponde a Cosas de la Vida: Sociedad, Gran Barcelona y Catalunya; y el tercero a Deportes. El cierre de este cuerpo lo ocupan las páginas de Lectores, Tiempo y Contraportada. Cada una de estas secciones cuenta con una portadilla en la que se adelantan, en sumarios, los temas más importantes de la misma.

El Periódico utiliza el color no sólo en las fotografías, sino también en los fondos con una clara función señalética. Así, los “apuntes”, un breve comentario de opinión respecto a los temas que abren las diferentes secciones van siempre ubicados sobre una trama de color. Además, en cada sección se emplea un color como leitmotiv: oro en "Tema del Día", azul en "Internacional", verde en "Política", morado en "Economía", naranja en "Cosas de la vida" y amarillo en "Deportes". Cada un de estos colores domina en los distintos elementos de las secciones -folio, tramas, cintillos, filetes- ayudando a mantener la continuidad visual de las mismas. Este planteamiento -habitual en algunos diarios norteamericanos, por ejemplo, USA Today- es completamente novedoso en la prensa española.

El segundo de los cuerpos de El Periódico, que va grapado y se encuentra dentro del principal, está formado por una revista diaria, Exit, que contiene las secciones de Espectáculos, Gente, El Día por Delante y Radio y Televisión. Este cuadernillo cuenta con su propia portada y está enfocado fundamentalmente al ocio y a los espectáculos.

Otras de las novedades llegadas con el rediseño reside en la oferta de suplementos temáticos semanales. A los ya existentes, como Viernes/Libros o Guía Teletodo se suman ahora otros dedicados a áreas como la economía, las nuevas tecnologías y la mujer: Ganar Más, Tecnológic@, Ella.

Se puede afirmar que el nuevo planteamiento formal de El Periódico responde claramente a la idea de diario de servicios. Esta concepción se refleja en varios aspectos. Por un lado, el propio diseño del rotativo está concebido para ayudar al lector a percibir de una manera sencilla los aspectos funcamentales de cada tema. En El Periódico se intenta que el número de historias a desarrollar en cada página sea mínimo, abundando las páginas monotemáticas. Esto permite abordar cada tema en profundidad y acompañarlo de despieces para la contextualización del mismo y de "Apuntes", breves textos de opinión en los que se ofrece un punto de vista que ayude a interpretar la información. En una tendencia que también recuerda a USA Today, El Periódico huye de la excesiva longitud en los textos de sus informaciones y reportajes. Se trata de textos más bien breves -rara vez superan los 2.500 caracteres-, donde la profundidad se ofrece en los elementos de apoyo.

El Periódico continúa otorgando una importancia primordial a la fotografía - a gran tamaño y en color- pero recurre con mucha frecuencia a los gráficos explicativos para ayudar al lector a visualizar datos, o para que pueda entender mejor cómo ha ocurrido algo. 


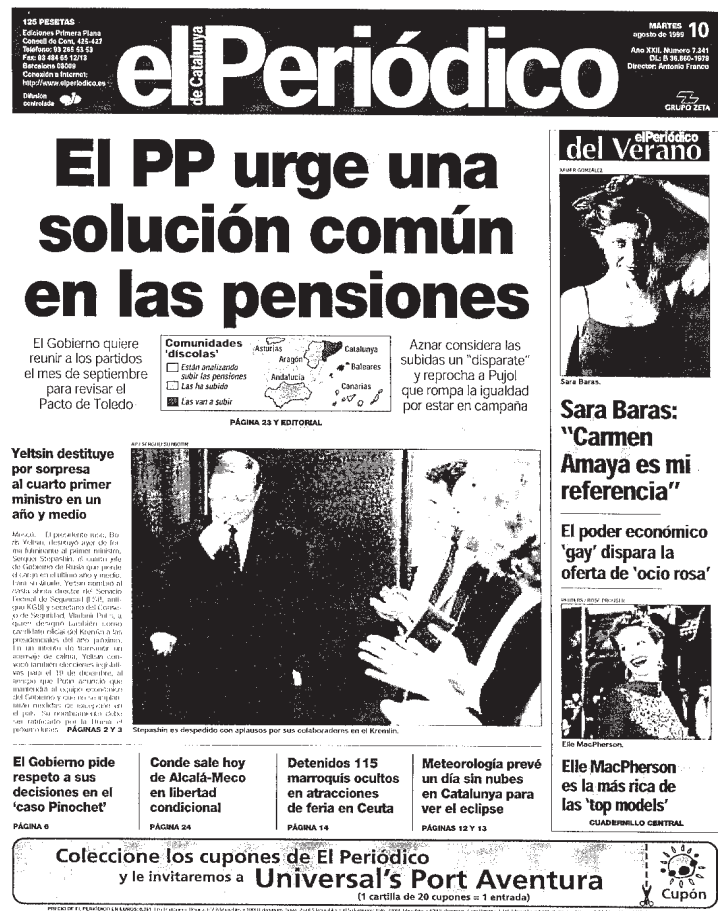

El Periódico de Catalunya renovó su imagen el 3 de diciembre de 2000. En la nueva confección (imagen inferior) la totalidad de las páginas de la publicación se imprimen en color.

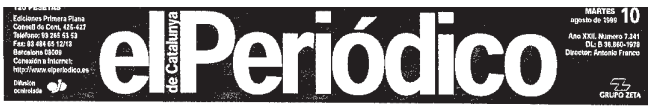

El PP urge una solución común en las pensiones
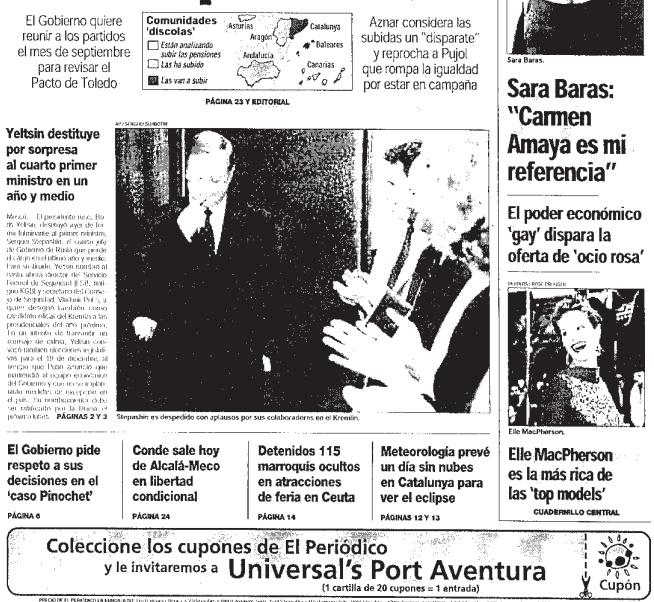
Dentro de este planteamiento de servicios no es de extrañar que uno de los géneros que más se ha reforzado tras el rediseño sea el informe. De hecho, $E l$ Periódico ha apostado por el área de reportajes y la ha dotado de estructura propia, estando integrada por seis periodistas, bajo la coordinación de Iosu de la Torre, uno de los subdirectores de la publicación.

Pero el planteamiento de diario de servicios no se reduce únicamente a los aspectos formales. Una de las secciones más importantes del rotativo es "Cosas de la vida", en la que se incluyen aquellos temas que, por su contenido, o por su ubicación geográfica puedan ser considerados más cercanos al día a día de los lectores. El reportaje y el informe son los géneros estrella de esta amplia sección: temas sociales como la inmigración, la salud, el medio ambiente, la comunicación y la cultura encuentran acomodo en "Cosas de la vida". Las historias geográficamente más cercanas -más locales- también se ubican aquí. Por otra parte, los servicios más ligados al ocio -desde la información sobre viajes, al comentario sobre un estreno cinematográfico- se agrupan en Exit, que además cuenta con una completísima agenda.

\subsection{Análisis comparativo de El Periódico de Catalunya}

3.2.1. NúMERO DE TEXTOS DE SERVICIO Y NO SERVICIO

\begin{tabular}{|l|r|r|}
\hline Número de Noticias & El Periódico (Antes) & El Periódico (Ahora) \\
\hline & 170,0 & 164,5 \\
\hline Noticias de servicios & 58,0 & 106,0 \\
\hline Noticias de servicios & $34,0 \%$ & $64,5 \%$ \\
\hline Noticias no servicios & 112,0 & 58,5 \\
\hline$\%$ Noticias no servicios & $66,0 \%$ & $35,5 \%$ \\
\hline Número de textos & 226,0 & 225,5 \\
\hline Textos servicios & & 136,5 \\
\hline$\%$ textos servicios & $38,5 \%$ & $60,5 \%$ \\
\hline Textos no servicios & & 89,0 \\
\hline$\%$ textos no servicios & 139,0 & $39,5 \%$ \\
\hline texto publicado & $61,5 \%$ & $68.139,5$ \\
\hline Cm/2 & & $48.592,5$ \\
\hline Cm/2 textos servicios & $58.306,0$ & $71,5 \%$ \\
\hline$\% \quad 32.026,0$ & 19547,0 \\
\hline Cm/2 textos no servicios & $55,0 \%$ & $28,5 \%$ \\
\hline$\%$ & & \\
\hline
\end{tabular}


De acuerdo con los datos, podemos decir rotundamente que existe inicialmente, después de su rediseño, una apuesta clara de El Periódico de Catalunya para potenciar entre sus páginas las noticias y los textos de servicios. A raíz de los cambios, el porcentaje de noticias de servicios pasa del $34 \%$ al $64,5 \%$, un incremento más que notable. Otro tanto sucede con los textos de servicios, que pasan del $38,5 \%$ al $60 \%$. Un porcentaje algo inferior al de las noticias que indica que este diario utiliza frecuentemente despieces informativos no de servicios.

Por el contrario, como es lógico, el porcentaje de noticias y textos de no servicios desciende notablemente y pasa del $66 \%$ al $35,5 \%$ en el caso de las noticias, y del $61,5 \%$ al $39,5 \%$ en el caso de los textos.

Un dato que hemos incorporado al estudio es también el del espacio total en centímetros cuadrados dedicado a los textos de servicios y a los de no servicios antes y después del rediseño. Es interesante esta comparación ya que, circunstancialmente, podría suceder que el número de noticias y textos fuera elevado, pero no así su espacio en centímetros cuadrados, ya que podría tratarse de textos breves, escasamente importantes, o simplemente complementarios de uno central que no sea de servicios.

De acuerdo con los datos de la tabla anterior podemos decir que el espacio en centímetros cuadrados dedicados antes a los temas de servicios suponía un 55\% del espacio total, una cifra que pasa al $71,5 \%$ después del rediseño, con lo que podemos concluir que los textos de servicios, en la actualidad, ocupan un lugar mucho más relevante en las páginas de ese medio de comunicación.

3.2.2. Formatos DE DOBle VElocidad EN LAS NOTICIAS DE SERVICIOS Y NO SERVICIOS

\begin{tabular}{|c|c|c|}
\hline & El Periódico (Antes) & El Periódico (Ahora) \\
\hline No noticias servicios & 58,0 & 106,0 \\
\hline Noticias con formatos & 15,0 & 72,0 \\
\hline$\%$ sobre el total & $26,0 \%$ & $68,0 \%$ \\
\hline Número de formatos & 24,0 & 125,5 \\
\hline No noticias no servicios & 112,0 & 58,5 \\
\hline Noticias con formatos & 25,0 & 40,0 \\
\hline$\%$ sobre el total & $22,5 \%$ & $69,5 \%$ \\
\hline No de formatos & 32,0 & 71,0 \\
\hline
\end{tabular}

En este apartado vamos a comparar la utilización de formatos de doble velocidad, llamados también formatos de lectura rápida, para comprobar así si 
desde la perspectiva de los aspectos más formales existe también una tendencia a profundizar en uno de los aspectos propios del periodismo de servicios.

De la tabla precedente podemos deducir, por un lado, tal y como ya hemos apuntado, el importante incremento del número de noticias de servicios de El Periódico de Catalunya tras su último rediseño. Por lo que respecta a la utilización de formatos de doble velocidad, que es lo que analizamos en el presente apartado, podemos constatar el importantísimo incremento en la utilización de formatos de lectura rápida en las noticias de servicios, que pasan de 15 noticias con formatos antes del rediseño, a 72 noticias después de los cambios, lo que representa un incremento porcentual del 26,0\% al 68,0\%. El incremento, como es lógico, se mantiene también en lo que respecta al número de formatos utilizados, que pasan de 24 antes del rediseño a 125,5 una vez que se han operado los cambios en este diario.

En lo que se refiere a las noticias de no servicios, constatamos también, como es lógico, el importante descenso habido en este tipo de noticias, que pasan de 112 antes de los cambios a 58,5. Por lo que respecta a la utilización de formatos de lectura rápida, el incremento es similar al habido en las noticias de servicios, ya que el porcentaje pasa del 22,5\% antes del rediseño al 69,5\% después del mismo. Este incremento se mantiene también, como es natural, en lo que se refiere al número de formatos utilizados, que pasan de 32 a 71 .

\subsubsection{LOS TEXTOS DE SERVICIOS Y SU ACTUALIDAD}

A continuación vamos a comparar si los textos de servicios que El Periódico de Catalunya publicaba antes de su rediseño se regían más o menos que ahora por la pauta de la actualidad periodística. Se trata de un dato interesante, ya que la vinculación de un texto a lo que entendemos por actualidad diaria, es un reflejo de la política informativa de los medios de comunicación.

Si un medio se aleja de lo que normalmente forma parte de la actualidad, entendida ésta como los hechos diariamente sucedidos, quiere decir que se preocupa por trabajar sus propios temas, informaciones diferenciadas de los medios de la competencia. Los datos comparativos podemos observarlos en la tabla anexa.

\begin{tabular}{|l|r|r|}
\hline & El Periódico (Antes) & El Periódico (Ahora) \\
\hline Textos de servicios & & \\
\hline No Textos de servicios $^{\circ}$ & 87,0 & 136,5 \\
\hline Textos de actualidad & 70,0 & 105,5 \\
\hline$\%$ no actualidad & 17,0 & $77,0 \%$ \\
\hline Textos no & 31,0 \\
\hline$\%$ & $19,5 \%$ & $23,0 \%$ \\
\hline
\end{tabular}


Por lo que respecta a la actualidad de los textos de servicios podemos decir que El Periódico de Catalunya mantiene una leve tendencia a trabajar más sus textos periodísticos, buscando sus propios temas y trabajándolos a través de sus propias fuentes, aunque en un porcentaje muy moderado, ya que el incremento de los textos de no actualidad va del $19,5 \%$ antes de su rediseño al $23 \%$ después de los cambios del diario. Por otra parte, el porcentaje de textos de servicios que se ajusta a la actualidad diaria es bastante elevado, ya que alcanza la cota del $77 \%$. Se constata pues un esfuerzo tenue del diario para buscar sus propios textos de servicios, aunque todavía se mantiene en unas cotas bastante bajas.

\subsubsection{LOS TEXTOS PUBLICADOS Y SUS GÉNEROS PERIODÍSTICOS}

\begin{tabular}{|l|r|r|}
\hline & El Periódico (Antes) & El Periódico (Ahora) \\
\hline $\mathbf{C m} / \mathbf{2}$ Totales & $58.306,0$ & $68.139,5$ \\
\hline $\mathrm{Cm} / 2$ informaciones & $49.953,0$ & $56.450,0$ \\
\hline$\%$ sobre el total & $85,5 \%$ & $83,0 \%$ \\
\hline $\mathrm{Cm} / 2$ reportajes & $1.403,0$ & $4.697,0$ \\
\hline$\%$ sobre el total & $2,5 \%$ & $6,5 \%$ \\
\hline & & $2.028,0$ \\
\hline $\mathrm{Cm} / 2$ entrevistas & $3.899,0$ & $3,0 \%$ \\
\hline$\%$ sobre el total & $6,5 \%$ & $3.451,5$ \\
\hline & & $5,0 \%$ \\
\hline $\mathrm{Cm} / 2$ de crónicas & $1.716,0$ & $1.253,0$ \\
\hline$\%$ sobre el total & $3,0 \%$ & $2,0 \%$ \\
\hline & & 260,0 \\
\hline $\mathrm{Cm} / 2$ de artículos & $1.200,0$ & $0,50 \%$ \\
\hline$\%$ sobre el total & $2,0 \%$ & \\
\hline
\end{tabular}

En este apartado vamos a comparar el uso que El Periódico de Catalunya hace de los géneros periodísticos antes y después de sus cambios. Para realizar el estudio hemos agrupado los textos periodísticos en tres bloques de géneros: géneros informativos, géneros interpretativos y géneros de opinión.

En el caso de los géneros informativos hemos recogido el género por excelencia: la información o noticia. En los géneros interpretativos hemos analizado los más representativos: el reportaje, la entrevista y la crónica. Por lo que respecta a los géneros de opinión hemos analizado los géneros más importantes que aparecen en las páginas de información general, es decir, el artículo de opinión y la crítica. 
Por lo que respecta a los géneros periodísticos utilizados por El Periódico de Catalunya antes y después de sus transformaciones formales, podemos decir que no existen cambios dignos de mención. Tanto antes como ahora, El Periódico de Catalunya utiliza en un altísimo porcentaje el género información, con un ligerísimo descenso, ni tan siquiera considerable, ya que va del $85,5 \%$ al $83 \%$.

Por lo que respecta a los géneros interpretativos, se incrementa en cuatro puntos la utilización de los reportajes y en dos puntos las crónicas, pero desciende tres puntos y medio en la utilización de entrevistas, por lo que en general los géneros interpretativos se mantienen en un nivel similar.

Los géneros de opinión, por su parte, no sufren cambios y tanto antes como ahora los artículos de opinión aparecen en un 2,0\% del total, mientras que las críticas se mantienen en la cota mínima del $0,5 \%$.

Los datos comparativos antes y después del rediseño de El Periódico de Catalunya quedan recogidos en la tabla anexa.

\subsubsection{Los elementos gráficos en El Periódico de Catalunya}

En este apartado vamos a analizar comparativamente el espacio en centímetros cuadrados que El Periódico de Catalunya dedicaba antes y dedica ahora a texto escrito y a material gráfico, para comprobar así la tendencia existente entre el material escrito y el gráfico. Asimismo, estudiaremos y compararemos el número de noticias que publicaba y publica con elementos gráficos y porcentaje sobre el total de noticias publicadas, para observar si la tendencia es publicar más o menos noticias con elementos gráficos. Los resultados comparativos aparecen en la tabla anexa.

\begin{tabular}{|l|r|r|}
\hline $\mathbf{C m} / 2$ Totales & El Periódico (Antes) & El Periódico (Ahora) \\
\hline & $58.306,0$ & $68.139,5$ \\
\hline Cm/2 de texto & $45.870,0$ & $42.470,5$ \\
\hline$\%$ sobre el total & $78,5 \%$ & $62,0 \%$ \\
\hline Cm/2 material gráfico & $12.436,0$ & $25.669,0$ \\
\hline$\%$ sobre el total & $21,5 \%$ & $38,0 \%$ \\
\hline No total de noticias & & 164,5 \\
\hline & 170,0 & 91,5 \\
\hline Noticias con gráficos & 71,0 & $55,5 \%$ \\
\hline$\%$ sobre el total & $42,0 \%$ & 146,5 \\
\hline & & 1,6 \\
\hline Número de gráficos & 88,0 & \\
\hline
\end{tabular}


Por lo que respecta al espacio que El Periódico de Catalunya dedicaba antes y ahora al material gráfico, podemos decir que existe una tendencia ascendente a ocupar cada vez más espacio con fotografías y otros elementos ilustrativos. Después de su rediseño, desciende bastante el espacio que ese diario dedica en sus páginas a informaciones escritas y el porcentaje pasa del 78,5\% antes del rediseño al $62 \%$ que utiliza en la actualidad. En esta misma proporción de 16,5 puntos se contabiliza el ascenso del espacio dedicado al material gráfico, que pasa del 21,5\% al $38 \%$. Existe pues una clara tendencia a utilizar muchas más informaciones gráficas que en la etapa anterior.

En este apartado de los elementos gráficos hemos comparado también el número de noticias con elementos gráficos que publica El Periódico de Catalunya. Así, antes de sus cambios este diario catalán utilizaba gráficos en el $42 \%$ del total de sus noticias publicadas, un porcentaje que sufre un notable incremento hasta la cota del 55,5\%, es decir 13,5 puntos por encima de la etapa anterior. Como consecuencia de estos datos podemos decir que, en la actualidad, El Periódico de Catalunya ilustra una de cada dos noticias, una cifra muy importante.

Finalmente, hemos comparado también el número de elementos gráficos que aparecen en cada noticia ilustrada, y el porcentaje asciende del 1,2 al 1,6; es decir, en la actualidad, en cada noticia ilustrada aparecen 1,5 ilustraciones. La tendencia actual en El Periódico de Catalunya no es la de ilustrar las noticias con un único elemento gráfico, sino que se tiende más bien a utilizar varios elementos gráficos para ilustrar las noticias.

Con anterioridad a su rediseño la tendencia de este periódico era la de utilizar una única ilustración en las noticias con elementos gráficos; sin embargo, en la actualidad se ha invertido esa tendencia y se tiende a utilizar más de una ilustración en las noticias con elementos gráficos, de ahí que se haya pasado del porcentaje del 1,2 al 1,6.

\subsubsection{SECCIONES CON MENOR y MAYOR PORCENTAJE DE SERVICIOS}

En este punto vamos a comparar el antes y ahora de las secciones en las que tradicionalmente no aparecen textos de servicios, y también el antes y ahora de las secciones que habitualmente publican textos de servicios, para comprobar así las tendencias de estos dos grupos.

Los datos de las secciones que tradicionalmente no publican informaciones de servicios están recogidos en la tabla anexa:

Tanto antes como después de los cambios habidos en El Periódico de Catalunya, las secciones con menor porcentaje de textos de servicio son las de Internacional, Política, Economía y Deportes; sin embargo, a través de los datos se constatan unos cambios que son representativos.

Las secciones de Internacional y Política, que antes nunca publicaban textos de servicios, publican ahora informaciones de este tipo en unos porcentajes del 
Secciones con menor porcentaje de textos de servicios

\begin{tabular}{|l|r|r|}
\hline & El Periódico (Antes) & El Periódico (Ahora) \\
\hline & & \\
\hline Secciones no servicios & & \\
\hline & $100,0 \%$ & $87,0 \%$ \\
\hline Internacional & $100,0 \%$ & $90,0 \%$ \\
\hline Política & $87,5 \%$ & $53,0 \%$ \\
\hline Economía & $100,0 \%$ & $100,0 \%$ \\
\hline Deportes & &
\end{tabular}

Secciones con mayor porcentaje de textos de servicios

\begin{tabular}{|l|r|r|}
\hline & El Periódico (Antes) & El Periódico (Ahora) \\
\hline & & \\
\hline Secciones servicios & & \\
\hline Tema del Día & $80,0 \%$ & $58,0 \%$ \\
\hline Cosas de la Vida & $31,0 \%$ & $65,5 \%$ \\
\hline Clasificados & $100,0 \%$ & $100,0 \%$ \\
\hline Ultima & $100,0 \%$ & $95,5 \%$ \\
\hline Sección servicios & $96,0 \%$ & $100,0 \%$ \\
\hline
\end{tabular}

$13 \%$ y $10 \%$, respectivamente. Estas dos secciones han comenzado a publicar pequeños textos explicativos, acompañados habitualmente de gráficos u otras ilustraciones, para explicar conflictos o situaciones complicadas en el ámbito de la política internacional o nacional.

Por lo que respecta a la sección de Economía, antes de los cambios publicaba algún texto de economía práctica, tal y como podremos comprobar posteriormente; sin embargo, el porcentaje del $13,5 \%$ de textos de servicios se debe fundamentalmente a la información de la Bolsa, un texto claro de servicio al lector. Sin embargo, en la actualidad, el porcentaje de textos de servicios en esta sección es del $47 \%$, una cota muy alta que se debe tanto a un notable incremento en la publicación de textos relacionados con lo que podemos denominar economía práctica, como por el incremento de información diaria dedicada a la Bolsa.

La única sección en la que no aparecen cambios es en la de Deportes. Tanto antes del rediseño como después, El Periódico de Catalunya nunca publica informaciones de servicio en esta sección.

Por lo que respecta a las secciones que publican un mayor porcentaje de textos de servicios, tenemos que hacer una aclaración inicial. En la actualidad, El Periódico de Catalunya publica una macrosección, toda ella de servicios, que lleva el nombre de Exit y que recoge lo que antes de su rediseño eran las secciones de 
Espectáculos, El Día x Delante, Cartelera y Radio y Televisión. Para poder realizar una comparación con la sección actual de Exit, hemos agrupado los resultados parciales de todas estas secciones en una única sección que hemos denominado Sección Servicios.

Los datos de la Sección Servicios correspondientes a antes del diseño corresponden, pues, a la suma de datos de las secciones citadas, mientras que el dato que aparece en la Sección Servicios en la columna de El Periódico (Ahora) corresponde a lo que actualmente se denomina Exit.

Los datos de las secciones que tradicionalmente publican más informaciones de servicios están recogidos en la tabla anexa.

Como podemos observar a través de los datos arriba apuntados, existen tres secciones en las que los porcentajes de textos de servicios antes y después del rediseño se mantienen inamovibles. Se trata de las secciones de Clasificados, Última y la que hemos denominado Sección Servicios para antes del rediseño y que se corresponde en la actualidad con la sección denominada Exit, todas ellas con un porcentaje del $100,0 \%$.

Los cambios más significativos los encontramos en la sección Tema del Día, en la que anteriormente se publicaban textos de servicios en un porcentaje del $80 \%$ y que en la actualidad ha descendido al 58\%. La explicación a esta circunstancia la podemos explicar no por el desinterés de El Periódico de Catalunya por los temas de servicio, sino que al no existir anteriormente una sección específica para este tipo de temáticas, acudían con mayor frecuencia a esta sección que abre el periódico. En la actualidad, sin embargo, al existir la sección de Exit, muchos temas que anteriormente debían publicarse de forma destacada en la de Temas del Día, encuentran ahora un perfecto acomodo en la nueva sección creada especialmente para esas temáticas.

Esta explicación parece lógica si tenemos en cuenta también el incremento habido en la publicación de temas de servicios en la sección de Cosas de la Vida. Una sección dedicada a temas de sociedad y que sufre un incremento en la utilización de informaciones de servicios que va del $31 \%$ antes del rediseño al $65,5 \%$ que utiliza en la actualidad.

\subsubsection{LAS TEMÁtiCAS DE SERVICIOS}

En este apartado vamos a analizar comparativamente las informaciones que sobre temas de servicios ha publicado El Periódico de Catalunya antes y después de sus importantes cambios formales. Para ello vamos a separar, al igual que hicimos con El Correo, el conjunto de temas de servicios en cuatro bloques, para distinguir lo que son noticias de servicios de los servicios que ofrecen los medios de comunicación al público.

La comparación general de todos los datos pertenecientes a estos cuatro apartados, tanto antes como después de su rediseño los encontramos en la tabla anexa: 


\begin{tabular}{|c|c|c|}
\hline Temáticas de servicio & Periódico (Antes) & Periódico (Ahora) \\
\hline Música, Cine, Literatura, Arte & $3.779,0$ & $4.978,0$ \\
\hline Salud & 560,0 & $1.993,5$ \\
\hline Acontecimientos Incidencia Directa & $2.084,0$ & $3.738,5$ \\
\hline Gente (Cotilleo) & $1.200,0$ & $2.225,0$ \\
\hline Solidaridad, (ONG). Inmigración & 600,0 & $1.950,0$ \\
\hline Ecología & $1.270,0$ & $1.152,0$ \\
\hline Educación & $1.232,0$ & 780,0 \\
\hline Ciencia & - & 917,0 \\
\hline Tecnología, Informática, Internet & 302,0 & $1.188,0$ \\
\hline Economía Práctica & 520,0 & $2.073,0$ \\
\hline Medios de Comunicación & 520,0 & 383,0 \\
\hline Consumo & - & 371,0 \\
\hline Varios & - & 219,0 \\
\hline Religiones & 460,0 & - \\
\hline Total Parcial & 12.527 .0 & $21.967,0$ \\
\hline \multicolumn{3}{|l|}{ Servicios al lector } \\
\hline Radio y Televisión & $4.256,0$ & $6.002,0$ \\
\hline Bolsa Valores & $2.128,0$ & $3.535,0$ \\
\hline Agenda & $2.563,0$ & 3.555 .5 \\
\hline Tiempo & 870,0 & 859,0 \\
\hline Sorteos & 194,0 & 205,0 \\
\hline Total Parcial & $10.011,0$ & $14.156,5$ \\
\hline \multicolumn{3}{|l|}{ Servicios-Ocio } \\
\hline Cine, Espectáculos (Cartelera) & $4.168,0$ & $7.016,0$ \\
\hline Pasatiempos & $1.064,0$ & $1.296,0$ \\
\hline Total Parcial & $5.232,0$ & $8.312,0$ \\
\hline \multicolumn{3}{|l|}{ Servicios-Comerciales } \\
\hline Esquelas & & 190,0 \\
\hline Clasificados & $4.256,0$ & $3.966,0$ \\
\hline Total Parcial & $4.256,0$ & $4.156,0$ \\
\hline Total Final & $32.026,0$ & $48.592,5$ \\
\hline
\end{tabular}

Dentro del primer apartado, que se refiere a las noticias de servicio y sus respectivas temáticas publicadas antes y después por El Periódico de Catalunya podemos observar un incremento constante en cada uno de los temas estudiados. Los incrementos más notables se producen en los apartados de Salud; Solidaridad, (ONGs), Inmigración; Tecnología, Informática, Internet y Economía Práctica. En cualquier caso, podemos decir que en general existe una política de profundizar en 
los temas que este diario ya venía apuntando claramente antes de sus cambios formales.

Si comparamos ahora el porcentaje que este tipo de noticias de servicios representan sobre la totalidad de los servicios publicados tendremos la tabla anexa:

De acuerdo con estos datos podemos observar cómo no solamente se incrementan los espacios dedicados a las noticias de servicios, sino que el incremento es también porcentual y pasa del 39,0\% al 45\%. Es decir, se incrementa porcentualmente el espacio que El Periódico de Catalunya dedica en su espacio a las informaciones de servicios respecto a la totalidad de servicios publicados.

También los servicios que El Periódico de Catalunya ofrecen a sus lectores se ven incrementados en datos relativos en todos y cada uno de sus apartados. Crece el espacio que dedica a la Radio y Televisión, aumenta también la Bolsa de Valores, así como el espacio dedicado a la Agenda. Se mantiene, sin embargo, el espacio que dedica al Tiempo y a los Sorteos.

Para analizar si se ha producido o no un incremento porcentual hemos elaborado la tabla siguiente:

Por lo que respecta a los servicios al lector debemos constatar que pese a que en la actualidad se han incrementado los centímetros cuadrados de espacio dedicado a los servicios al lector respecto a la etapa anterior, porcentualmente el espacio dedicado ahora a ese tipo de temas es algo menor que el que dedicada $E l$ Periódico de Catalunya ante de su rediseño.

Los servicios de ocio al lector ofrecidos por El Periódico de Catalunya se han visto notablemente incrementados en lo que respecta a la Cartelera de Espectáculos, que pasa de 4.168 centímetros cuadrados a 7.016. Sin embargo, aunque con una ligerísima tendencia ascendente, se mantienen los Pasatiempos con un espacio dedicado antes de 1.064 centímetros cuadrados y de 1.296 que dedica en estos momentos.

Para analizar si se ha producido o no un incremento porcentual hemos elaborado la tabla siguiente:

A pesar de que se incrementa notablemente el espacio de la Cartelera, porcentualmente, respecto a la totalidad de los servicios ofrecidos, se mantiene a un nivel similar al que tenía antes de los cambios. En su etapa anterior El Periódico de Catalunya dedicaba el 16,5\% del espacio de servicios a temas de ocio, un porcentaje que en la actualidad es del 17\%. Podemos decir, por consiguiente, que tras su rediseño El Periódico de Catalunya dedica porcentualmente a los temas de ocio un espacio similar al de su etapa anterior.

Por lo que respecta a los servicios comerciales, las esquelas sufren un ligero ascenso respecto a la etapa anterior, aunque se mantiene en unos porcentajes aún muy bajos. Por su parte, los anuncios clasificados sufren un ligero descenso, nada considerable y que hace que podamos decir que la tendencia en los servicios comerciales, aunque es en la actualidad un poco menor, se mantiene. 


\section{Conclusiones}

1.- Noticias de servicios y no servicios: Existe inicialmente, después de su rediseño, una apuesta clara de El Periódico de Catalunya para potenciar entre sus páginas las noticias y los textos de servicios. A raíz de los cambios, el porcentaje de noticias de servicios pasa del $34 \%$ al $64,5 \%$. Otro tanto sucede con los textos de servicios, que pasan del $38,5 \%$ al $60 \%$. Un porcentaje algo inferior al de las noticias que indica que este diario utiliza frecuentemente despieces informativos no de servicios.

2.- Tipos de formatos de doble velocidad en las noticias de servicio y no servicio: Por lo que respecta a la utilización de formatos de doble velocidad podemos constatar el importantísimo incremento en la utilización de formatos de lectura rápida en las noticias de servicios, que pasan del 26,0\% al 68,0\%. En lo que se refiere a las noticias de no servicios el incremento es similar al habido en las noticias de servicios, ya que el porcentaje pasa del 22,5\% antes del rediseño al $69,5 \%$ después del mismo.

3. Los textos de servicios y su actualidad: Por lo que respecta a la actualidad de los textos de servicios podemos decir que El Periódico de Catalunya mantiene una leve tendencia a trabajar más sus textos periodísticos, buscando sus propios temas y trabajándolos a través de sus propias fuentes, aunque en un porcentaje muy moderado, ya que el incremento de los textos de no actualidad va del 19,5\% antes de su rediseño al $23 \%$ después de los cambios del diario.

4.- Los textos publicados y los géneros periodísticos: En lo que se refiere a los géneros periodísticos utilizados antes y después del rediseño de El Periódico de Catalunya podemos decir que no existen cambios dignos de mención. Tanto antes como ahora, El Periódico de Catalunya utiliza en un altísimo porcentaje el género información, con un ligerísimo descenso, ni tan siquiera considerable, ya que va del $85,5 \%$ al $83 \%$. Por lo que respecta a los géneros interpretativos, se incrementa en cuatro puntos la utilización de los reportajes y en dos puntos las crónicas, pero desciende tres puntos y medio la utilización de entrevistas, por lo que en general los géneros interpretativos se mantienen en un nivel similar. Los géneros de opinión, por su parte, no sufren cambios y tanto antes como ahora los artículos de opinión aparecen en un 2,0\% del total, mientras que las críticas se mantienen en la cota mínima del $0,5 \%$.

5.- Los elementos gráficos: Por lo que respecta al espacio que El Periódico de Catalunya dedicaba antes y ahora al material gráfico, podemos decir que existe una tendencia ascendente a ocupar cada vez más espacio con fotografías y otros elementos ilustrativos. Después de su rediseño, el espacio dedicado al material gráfico pasa del $21,5 \%$ al $38 \%$, por lo que podemos decir que existe pues una clara tendencia a utilizar muchas más informaciones gráficas que en la etapa anterior.

6.- Secciones con menor y mayor porcentaje de textos de servicios: Tanto antes como después de los cambios habidos en El Periódico de Catalunya, las 
secciones con menor porcentaje de textos de servicio son las de Internacional, Política, Economía y Deportes.

Por lo que respecta a las secciones con mayor porcentaje de textos de servicios, existen tres secciones en las que los porcentajes de textos de servicios antes y después del rediseño se mantienen inamovibles. Se trata de las secciones de Clasificados, Última y la que hemos denominado Sección Servicios para antes del rediseño y que se corresponde en la actualidad con la sección denominada Exit, todas ellas con un porcentaje del 100,0\%.

7.- Las temáticas de servicios: En lo que se refiere a las noticias de servicios, podemos observar un incremento constante en cada uno de los temas estudiados. Los incrementos más notables se producen en los apartados de Salud; Solidaridad, (ONGs), Inmigración; Tecnología, Informática, Internet y Economía Práctica.

Si comparamos ahora las noticias de servicios con la totalidad de servicios ofrecidos por El Periódico de Catalunya tenemos que, en la actualidad, el espacio dedicado a noticias de servicios y la suma de todos los servicios se han ido equilibrando y el porcentaje es del $45 \%$ de noticias y el $54 \%$ de servicios al lector. Una diferencia que deja bien a las claras la importancia que para El Periódico de Catalunya tienen las noticias de servicios, que van ganando terreno a lo que son servicios que el diario ofrece a los lectores.

\section{$\underline{\text { Referencias bibliográficas: }}$}

- ARMENTIA/ELEXGARAY/PÉREZ (1999): Diseño y periodismo electrónico. UPV. Leioa.

- ARMENTIA VIZUETE, J.I. y CAMINOS MARCET, J.M. (1998): La información. Redacción y estructuras. Universidad del País Vasco. Leioa.

- BENESH, Susan (1998): «The Rise of Solutions Journalism». In Columbia Journalism Review. Marzo/abril 1998. En http://www.cjr.org/year/98/2/ solutions.asp.

- CASASÚS, José María y NÚNEZ LADEVÉZE, Luis (1991): Estilo y géneros periodísticos. Ariel Comunicación. Barcelona.

- CASASÚS, J.M. y otros (1991): La organización redaccional del nuevo diseño periodístico. La Vanguardia. Barcelona.

- DADER, José Luis: «La recuperación ciudadana de los medios (Vías de participación y contrapeso crítico de los consumidores y usuarios ante los medios de comunidación de masas». Noticias de Comunicación. In http:// comunicacion.boschce.es/articulos/nc018_02.htm

- DIEZHANDINO, Pilar (1994): Periodismo de servicio. Bosch. Barcelona. 
- EIDE, Martin y KNIGHT, Graham (1999). «Public/Private Service. Service journalism and the problems of every day life». European Journal of Communication. Vol 14 (4): Págs.525-547.

- FONTCUBERTA, Mar de (2000): «Educación y comunicación en los albores del siglo XX». In Reflexiones Académicas. $\mathrm{N}^{\circ}$ 10. In http://facom.udp.cl/CEM/ grl/educa/fontcu.htm

- LIPOVETSKY, Gilles (2000): La era del vacío. Anagráma. Barcelona.

- LEMMON, Jack: «The view of service journalism». In First Forum of the Committee of Concerned Journalists. In http://www.journalism.org/Report3.html

- LIBERMAN, Trudy (1994): «Whatever happened to consumer reporting?»In Columbia Journalism Review. Sept/octubre de 1994. In http://www.cjr.org/year/ 94/5/consumer.asp

- MERCHÁN, Iker (2000): «Análisis del cambio de diseño en el diario El Correo». In Coranto.net. Disponible en http://www.coranto.net

- MERRITT, Davis (1995): Public Journalism and Public Life: Why Telling the News is not Enough. Hillsdale. Erlbaum. N.J.

- METZLER, Ken (1986): Newsgathering. Prentice-Hall Inc. New Yersey.

- MEYER, Philip. (1996): Periodismo de precisión. Nuevas fronteras para la investigación periodística. Bosch. Barcelona.

- MEYER, Philip (1986): «El periodismo de servicio público y el problema de la objetividad». In http://www.terra.es/personal/f.casal/PAO/meyer.htm.

- Meredith Corporation 2000. Annual Report. In http://www.meredith.com/investors/annreprt.html

- MORIN, Edgar (1997): Une politique de civilisation. Arlea. París.

- Project (The) for Excellence in Journalism and the Medill News Service Washington Bureau. Changing definittions of news. Is there really a trend toward entertainment as news? If there is, what form does it take? In http:// www.journalism.org/lastudy.html

- RANLY, Don (1998): «Service Journalism: Presenting Useful Information in the Most Usable Way». Ver en http://www.adventist.org/aei/news/conference/ ranly3html

- ROSEN, Jay Art: «El periodismo público y los problemas de la prensa». Ensayo del Twentieth Century Fundation. Disponible en http://www.civnet.org./vitas/ panam/rosen $1 /$ rosen.1.htm

- ROSEN, Joe and MERRITT, Davis (1994): Public Journalism. Theory and Practice. Kettering Foundation. Ohio.

- RUIZ, Juan Cruz (2000): El peso de la fama. El País. Madrid.

- WERNICK, Andrew (1991): Promotional Culture: Advertising, Ideology and Symbolic Expression. Sage. London. 